\title{
Sub-femtosecond electron bunches in laser wakefield acceleration via injection suppression with a magnetic field
}

\author{
Q. Zhao ${ }^{1,2}$, S. M. Weng ${ }^{1,2, \dagger}$, M. Chen ${ }^{1,2}$, M. Zeng ${ }^{3}$, B. \\ Hidding $^{4,5}$, D. A. Jaroszynski ${ }^{4,5}$, R. Assmann ${ }^{3}$, Z. M. Sheng ${ }^{1,2,4,5, \ddagger}$ \\ ${ }^{1}$ Key Laboratory for Laser Plasmas (MoE), Department of Physics and Astronomy, \\ Shanghai Jiao Tong University, Shanghai 200240, China \\ ${ }^{2}$ Collaborative Innovation Center of IFSA, Shanghai Jiao Tong University, Shanghai \\ 200240, China \\ ${ }^{3}$ Deutsches Elektronen-Synchrotron DESY, 22607 Hamburg, Germany \\ ${ }^{4}$ SUPA, Department of Physics, University of Strathclyde, Glasgow G4 0NG, UK \\ ${ }^{5}$ Cockcroft Institute, Sci-Tech Daresbury, Cheshire WA4 4AD, UK \\ E-mail: ${ }^{\dagger}$ wengsuming@gmail.com, or ${ }^{\ddagger}$ z.sheng@strath.ac.uk
}

\begin{abstract}
It is shown that electron injection into a laser-driven plasma bubble can be manipulated by applying an external magnetic field in the presence of a plasma density gradient. The down-ramp of the density-tailored plasma locally reduces the plasma wave phase velocity, which triggers injection. The longitudinal magnetic field dynamically induces an expanding hole in the electron density distribution at the rear of the wake bubble, which reduces the peak electron velocity in its vicinity. Electron injection is suppressed when the electron velocity drops below the phase velocity, which depends on the size of the density hole. This enables the start and end of electron injection to be independently controlled, which allows generation of sub-femtosecond electron bunches with peak currents of a few kilo-Ampere, for an applied magnetic field of $\sim 10$ Tesla.
\end{abstract}

PACS numbers: 52.38.Kd, 52.65.Rr

Submitted to: Plasma Phys. Control. Fusion

\section{Introduction}

A plasma density wave or wake driven by the ponderomotive force of an ultraintense laser pulse can trap electrons and accelerate them to high energies [1, 2]. This wellknown laser wakefield acceleration (LWFA) promises compact sources of high energy electrons because of the ultra-strong accelerating electric fields, which can exceed 100 $\mathrm{GV} / \mathrm{m}$, in the so-called bubble regime that is characterized by a spherical electron cavity containing ions and surrounded by a high-density electron sheath $[3,4,5,6]$. In particular, the electron bunches obtained in the LWFA can be ultrashort, which is 
a major advantage of the LWFA and of great significance as drivers of ultrashort Xray sources and potential compact $\mathrm{X}$-ray free-electron lasers $[7,8,9,10,11,12,13]$. In general, the high-quality electron bunch should reside within the accelerating and focusing region of a wakefield with a length about $\lambda_{p} / 4$ in the linear regime $[14,15]$, where $\lambda_{p}$ is the linear plasma wavelength. In the nonlinear bubble regime, the relativistic plasma wavelength $\lambda_{N} p$ for a highly relativistic laser intensity can be much longer than $\lambda_{p}[2]$. On the other hand, the longitudinal electric field will be highly steep around the bubble rear [5]. In this bubble regime, therefore, the injected bunch is also required to reside in a length as short as $\lambda_{p} / 4$ in order to reduce the energy spread. It is therefore expected that ultrashort electron bunches generated by a typical LWFA will have durations $\lambda_{p} / 4 c \sim 10$ femtoseconds for plasma densities $n_{e} \simeq 10^{18} \sim 10^{19}$ $\mathrm{cm}^{-3}$. There is considerable interest in generating even shorter electron bunches, with attosecond durations, for various applications including attosecond X-ray sources and direct imaging $[16,17,18,19]$.

In LWFA, a shorter bunch duration down to sub-femtosecond is possible if the injection of electrons into the wake is highly localized. The localized electron injection can be achieved by near-threshold self-injection [20, 21, 22], by colliding pulse injection $[14,15]$, by up-ramp density transition [23], or by self-truncated ionization injection $[24,25]$. Alternatively, the localized electron injection can be realized by manipulating the plasma wake structure. For instance, the local plasma wake phase and wavelength can be tuned by longitudinal plasma density tailoring [26]. To facilitate electron injection, the wake phase velocity can be reduced momentarily by a density down-ramp $[27,28,29,30,31,32,33,34]$. In particular, the highly localized injection can be achieved if the time in which the peak forward-directed plasma electron velocity exceeds the wake phase velocity is ultrashort, which promises the generation of attosecond electron bunches [34]. So far, the generation of isolated sub-femtosecond electron bunches has not yet been demonstrated experimentally and several technical challenges still need to be overcome.

It is well known that the wakefield structure in the LWFA can be modified by a static external magnetic field. This may provide an alternative approach to control the electron injection $[35,36,37,38,39,40]$. Due to the suppression of the electron transverse motion, it was found that the electron injection can be enhanced by a static longitudinal magnetic field of a few tens of tesla [36]. By imposing an external transverse magnetic field that is on the order of hundreds of Tesla, the longitudinal trapping condition in the selfinjection regime can be significantly relaxed [37], which also enhances the charge number of injected electrons. In contrast, it has been recently found that the external magnetic field required to modify the transverse trapping condition in the ionization-injection regime is only on the order of tens of Tesla [40], which promises efficient generation of high-quality electron bunches with both high charge and low energy spread. It is noted that strong magnetic fields of a few tens of Tesla can be generated in a small volume by either traditional technology in laboratories [41, 42] or a novel proposal using twisted laser beams [43]. 
In this paper, we present a study of the manipulation of the laser-driven plasma bubble to control the persistence of electron injection, through combining a densityprofile-tailored plasma with a longitudinal magnetic field. We show that the static longitudinal magnetic field modifies the transverse structure of the bubble, while the density gradient changes its longitudinal structure. The magnetic field induces a radial density hole in the bubble rear [38, 39], which expands and as the bubble evolves along the density down-ramp. Electron injection is triggered by the decreasing phase velocity of the bubble along the density down-ramp, and then suppressed by the expanding hole at the bubble rear. In this way, the position and persistence of electron injection can be controlled, leading to injection of isolated sub-femtosecond electron bunches.

\section{Theoretical analysis}

We first consider the effect of the plasma density gradient on the laser-driven plasma wake. In a tenuous inhomogeneous plasma, the wake wave has a local phase $\chi=k_{p}(z) \xi$, where $\xi=z-v_{p} t$ is the relative coordinate, $\omega_{p}=\left(n_{e} e^{2} / \varepsilon_{0} m_{e}\right)^{1 / 2}$ and $k_{p}=\omega_{p} / v_{p}$ are the local plasma frequency and wavenumber, respectively. For a non-relativistic laser pulse, the wavelength $\lambda_{p}(z)$ only depends on the local plasma density $n_{e}(z)$, and therefore $\beta_{p}=\beta_{g}\left(1+(\chi / 2 \pi)\left(d \lambda_{p} / d z\right)\right)^{-1}$, where $\beta_{p}=v_{p} / c$ and $\beta_{g}=v_{g} / c$ are the normalized wake phase and laser group velocities, respectively. For a relativistic laser pulse with initial peak normalized potential amplitude $a_{0} \gg 1$, the effect of laser amplitude evolution on $k_{p}(z)$ and $\omega_{p}(z)$ must be considered appropriately. In this case, the ponderomotively expelled electrons oscillate transversely at the relativistic betatron frequency $\omega_{\beta}=\omega_{p} / \sqrt{2 \gamma_{e}}$ with the Lorentz factor $\gamma_{e} \simeq \sqrt{1+a^{2} / 2}$ in the ponderomotive approximation [44]. The initially stationary electrons return to the laser axis after half a betatron oscillation period $\pi / \omega_{\beta}$ and cross at the bubble rear. The velocity of the bubble rear, or the phase velocity at $\chi=-2 \pi$, where the longitudinal electric field $E_{z}=0$, can be formulated as $\beta_{p}^{-1}=\beta_{g}^{-1}+c d \tau / d z$ [34], where $\tau=2 \pi / \omega_{\beta}$. This approximately gives the bubble velocity as

$$
\beta_{p}=\beta_{g}\left[1-\beta_{g} \frac{\lambda_{p 0}}{4}\left(\frac{\sqrt{\gamma_{e}}}{\tilde{n}^{3 / 2}} \frac{d \tilde{n}}{d z}-\frac{1}{\sqrt{\tilde{n} \gamma_{e}}} \frac{d \gamma_{e}}{d z}\right)\right]^{-1},
$$

where $\tilde{n}=n_{e} / n_{0}$ is the plasma density normalized to the plateau density $n_{0}$, and $\lambda_{p 0}$ is the plasma wavelength corresponding to $n_{0}$. The effects of the plasma density gradient and laser amplitude evolution are included in the terms $d \tilde{n} / d z$ and $d \gamma_{e} / d z$, respectively. The bubble velocity can be reduced by decreasing the plasma density and/or increasing the laser amplitude.

Under a strong longitudinal magnetic field $B_{0}$, however, the bubble rear will open up $[38,39]$. In this case, the electrons moving along the bubble sheath experience a time-varying magnetic flux $\phi=B_{0} \pi r_{b}^{2}(\xi)$, where $r_{b}$ is the radius of the bubble. This induces an azimuthal electric field, which causes the sheath electrons to obtain an azimuthal velocity $v_{\varphi}$ and rotate reversely around the laser axis. As a consequence, a huge longitudinal magnetic field $B_{z}$ is self-generated and distributes locally inside 
the density-hole region, with the same direction as $B_{0}$, as described by Lenz's law [39]. Finally, the radial motion of sheath electrons is governed by

$$
\frac{\partial p_{r}}{\partial t}+v_{r} \frac{\partial p_{r}}{\partial r}=\frac{p_{\varphi} v_{\varphi}}{r}-e W_{\perp}-e v_{\varphi}\left(B_{0}+B_{z}\right)
$$

where $p_{\varphi} v_{\varphi} / r$ is the centrifugal force, and $W_{\perp}=E_{r}-c B_{\varphi}$ is the radial wakefield $[5,6]$. It is this centrifugal force that opens up the bubble rear, because it tends to infinity when $r \rightarrow 0$. Considering the equations for the transverse momentum only, the radius of the hole in the opened bubble rear should be governed mainly by the plasma density and magnetic field, which can be approximated by $r_{\min } \approx 2 \sqrt{2}\left(c \omega_{c}\right) / \omega_{p}^{2}[38]$, where $\omega_{c}=e B_{0} / m_{e}$ is the electron cyclotron frequency. Nevertheless, since electrons experience a strong longitudinal acceleration in the wakefield, the hole radius also depends on the instantaneous mean electron energy around the bubble rear, because the centrifugal force $p_{\varphi} v_{\varphi} / r=m_{e} \gamma_{e} v_{\varphi}^{2} / r \propto \gamma_{e}$.

\section{PIC simulations}

To demonstrate the combined effects of plasma density tailoring and the longitudinal magnetic field on the wake structure, and therefore the consequent electron injection, three-dimensional (3D) particle-in-cell (PIC) simulations using OSIRIS [45] have been performed. In each simulation, a $30 \times 30 \times 70 \mu \mathrm{m}^{3}$ simulation box moves along the z-axis at the speed of light. It is subdivided into $120 \times 120 \times 2240$ cells with $1 \times 1 \times 2$ particles per cell. The plasma comprises a plateau background density $n_{0}$ corresponding to a plasma frequency $\omega_{p 0}$ and a Gaussian density bump

$$
\frac{n_{e}}{n_{0}}=1+\alpha \exp \left(-\left(z-z_{i}\right)^{2} / 2 \sigma_{z}^{2}\right),
$$

where $\alpha$ is the relative amplitude of the density peak at $z_{i}, \sigma_{z}$ is the characteristic length of the Gaussian bump. Such a plasma density profile is realizable in experiments as longs as the ramp does not need to be too steep [29, 46]. A linearly polarized (along y-direction) laser pulse with duration of $30 \mathrm{fs}$, with an initial peak normalized amplitude $a_{0}=4$ and a waist of $15 \mu \mathrm{m}$ at focal plane $z=0$, is used. The laser wavelength is $0.8 \mu \mathrm{m}$, correspondingly the critical plasma density $n_{c}=1.7 \times 10^{21} \mathrm{~cm}^{-3}$. A uniform external magnetic field $B_{0}$ is assumed to be along the $z$-axis and is exerted on the whole plasma region.

\subsection{Evolution of phase velocity due to bubble stretch}

Figure 1(a) displays the local plasma density and the laser amplitude as functions of the propagation distance $z$. It is seen that the laser amplitude decreases slightly along almost the whole density bump region before $\mathrm{z}=200 \mu \mathrm{m}$ and then increases rapidly because of self-focusing. Substituting into Eq. (1), the local wake phase velocity can be calculated analytically. Figure 1(b) illustrates that the bubble rear velocity decreases dramatically due to the decreasing plasma density at the down-ramp around $z \sim 200$ 

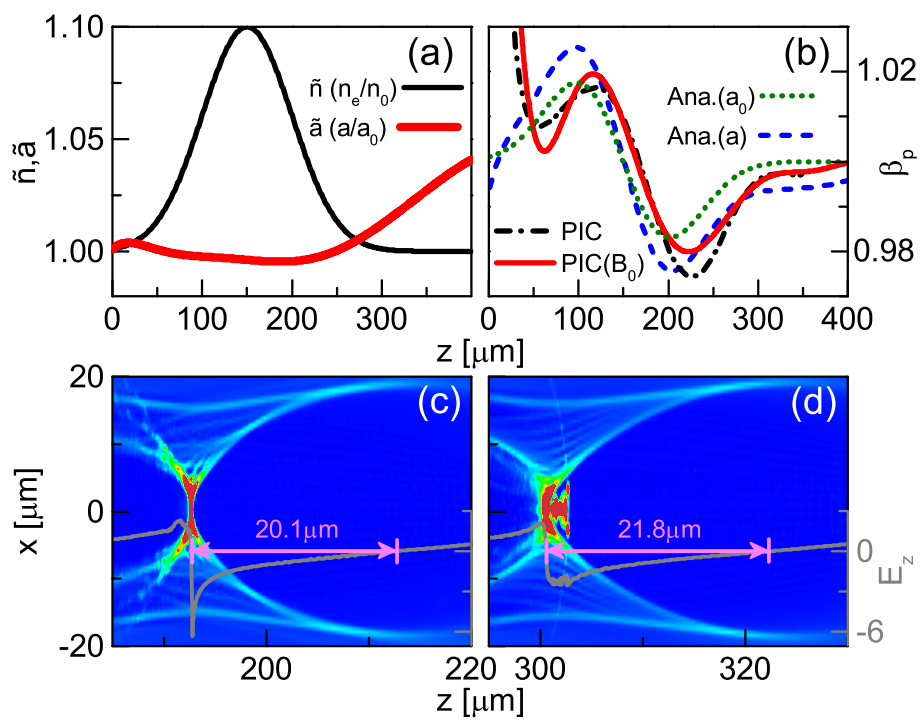

Figure 1. (Colour online) (a) Tailored plasma density profile with down-ramp parameters: $n_{0}=0.0005 n_{c}, z_{i}=150 \mu \mathrm{m}, \alpha=0.1$, and $\sigma_{z}=50 \mu \mathrm{m}$. In-situ laser amplitude obtained from the simulation, where $a_{0}=4$. (b) Bubble velocities obtained from Eq. (1) using $n_{e}$ with a constant $a_{0}$ (dot curve) and evolving $a$ as shown in (a) (dashed curve), and the simulations with $B_{0}=0$ (dash-dot curve) and $B_{0}=50 \mathrm{~T}$ (solid curve), respectively. Plasma bubble structures superimposed with the on-axis $E_{z}$ (normalized to $m_{e} c \omega_{p 0} / e$ ) in the case with $B_{0}=0$, when the bubble rear locates at (c) the slope $\left(z_{b}=192.7 \mu \mathrm{m}\right)$ and $(\mathrm{d})$ the bottom $\left(z_{b}=300.7 \mu \mathrm{m}\right)$ of the down-ramp, respectively.

$\mu \mathrm{m}$. The simulation result is in good agreement with the analytical estimation by Eq. (1) using a varying laser amplitude $a$ extracted from PIC simulations. The bubble rear velocity estimated by Eq. (1) using a constant $a_{0}$ is also drawn for comparison. The reduction in the bubble velocity is mainly attributed to the increase of the wavelength of the wake. The longitudinal stretch of the wake bubble is confirmed in figures 1(c) and $1(\mathrm{~d})$, where the radius of the bubble increases from 20.1 to $21.8 \mu \mathrm{m}$. As a consequence, electron injection is triggered, as is illustrated in figure 1(d). From figure 1(b), one notes that the reduction in the bubble velocity is slightly weakened by a longitudinal magnetic field $B_{0}=50 \mathrm{~T}$. This is because the injected electron bunch in the $B_{0}=0$ case causes a strong beam loading effect, which enhances the bubble elongation and reduces the bubble velocity further. In contrast, the injected bunch charge is significantly reduced in the $B_{0}=50 \mathrm{~T}$ case (will be discussed in more detail in the next section) and hence the beam loading effect is much weaker in this case. It is worth pointing out that the electron bunch length, transverse emittance, charge number could be well controlled in density-gradient injection by varying the density difference and steepness of the density down-ramp [30, 31]. 

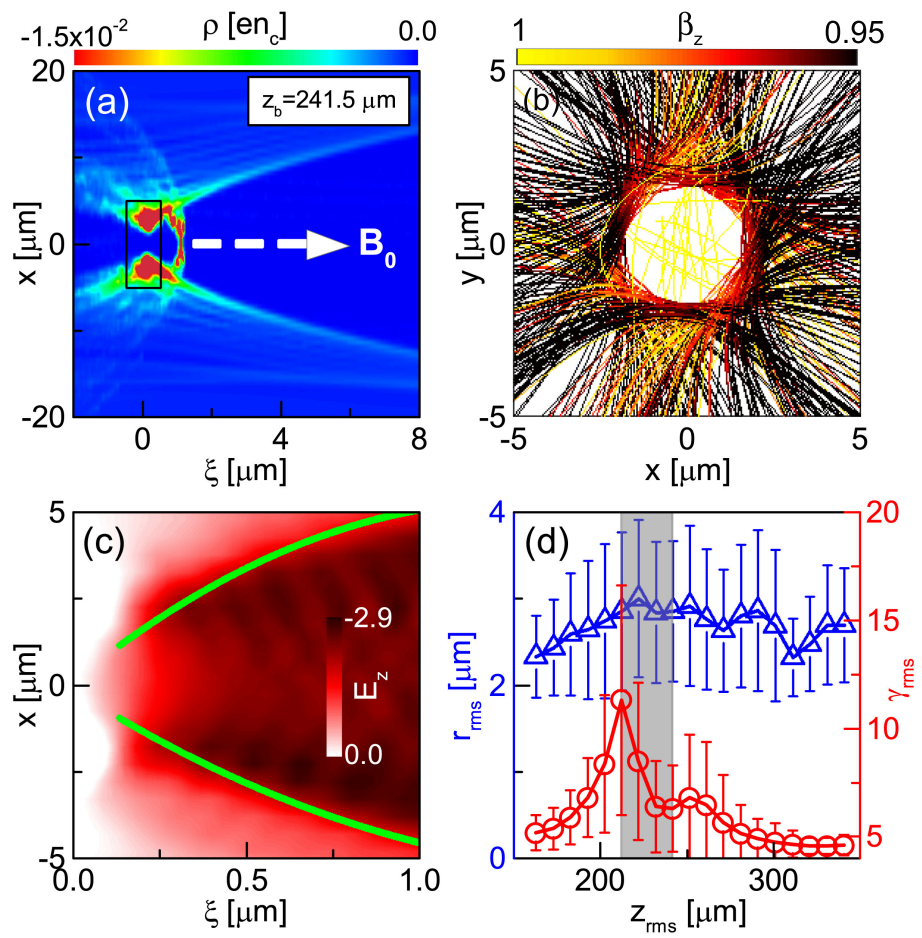

Figure 2. (Colour online) (a) Bubble structure for the bubble rear located at $z_{b}=214.5 \mu \mathrm{m}$, where $\xi=z-v_{p} t$. (b) Trajectories of 500 electrons in the $x-y$ plane, the electrons are randomly selected from the rectangle region in (a) and the color denotes their instantaneous longitudinal velocities. (c) $E_{z}$ corresponding to the bubble in (a). Green lines outline the bubble sheath. (d) Time evolution of the rootmean-square (RMS) radius $r_{\mathrm{rms}}$ and the Lorentz factor $\gamma_{\mathrm{rms}}$ of energetic electrons around the bubble rear, where their RMS distributions are given. Grey shaded region marks the space where electron injection occurs. Except for $B_{0}=50 \mathrm{~T}$, all other parameters are the same as those in figure 1.

\subsection{Magnetic field induced injection suppression along a density down-ramp}

To understand the effect of a non-zero $B_{0}$ on the electron injection, it is important to reveal first its effect on the bubble structure. As previously predicted [38, 39], figure 2(a) confirms the appearance of an open bubble rear for $B_{0}=50 \mathrm{~T}$. More importantly, the electron injection in this case is found to take place over a short distance. The electron bunch $(\sim 0.5 \mu \mathrm{m})$ resulting from this highly localized injection is much shorter than that $(\sim 2 \mu \mathrm{m})$ in the case with $B_{0}=0$. Figure $2(\mathrm{~b})$ displays the trajectories of 500 electrons in the transverse $\mathrm{x}-\mathrm{y}$ plane, in which the electrons are randomly selected from the rectangle region in Fig. 2(a). As predicted by the theoretical analysis, the electrons moving along the bubble sheath under a longitudinal magnetic field will obtain azimuthal velocities due to the azimuthal electric field induced by a time-varying magnetic flux. As a consequence, these electrons will be defected outward at an off-axis radius by the strong centrifugal force and form a hole at the bubble rear. Furthermore, Figure 2(b) clearly shows that only a few of electrons can approach the axis and be injected. This confirms that the electron injection has been almost terminated at $z_{b}=241.5 \mu \mathrm{m}$. 

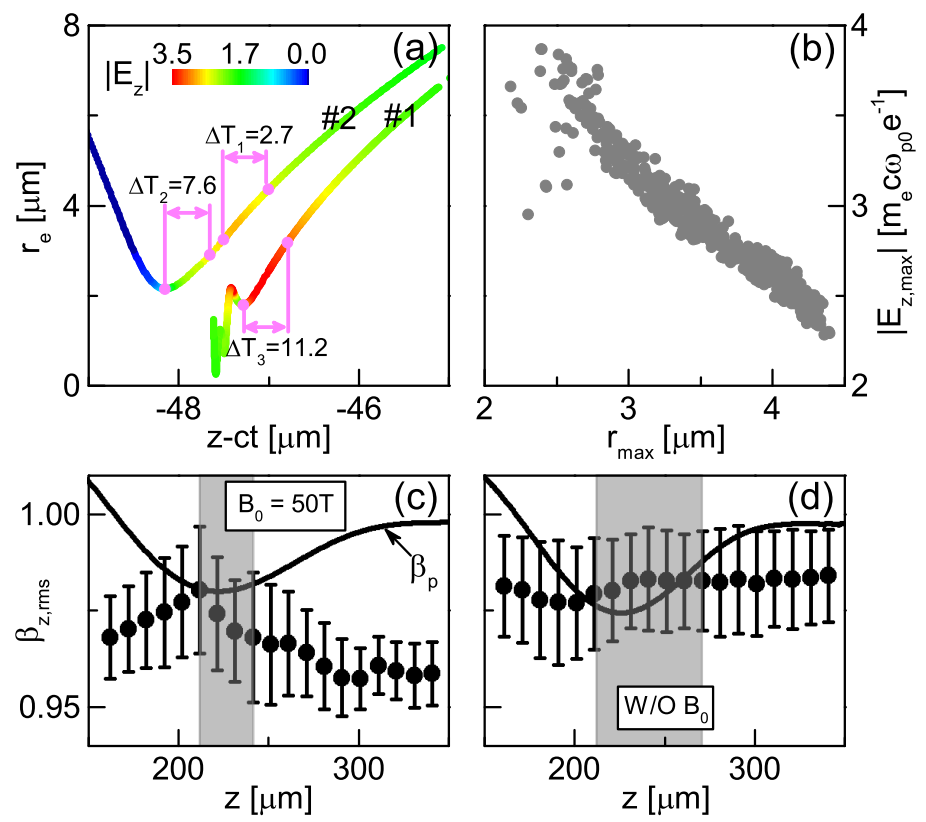

Figure 3. (Colour online) (a) Typical electron trajectories selected from the injected bunch $(\# 1)$ and the unclosed bubble rear (\#2) in figure 2(a), respectively. Different standing time in some typical acceleration regions (with the same $\Delta(z-c t)=0.5$ $\mu \mathrm{m})$ are marked as $\Delta T_{1,2,3}$. Time is normalized to $1[\mu \mathrm{m}] / c$. (b) Correlation between the maximums of $E_{z}$ and the radial positions where these maximums are achieved for 500 electrons randomly selected from figure 2(b). The RMS longitudinal velocity $\beta_{z, r m s}$ with its RMS distribution for the energetic electrons at the bubble rear vs the simulated local bubble velocity $\beta_{p}$ along the density down-ramp with (c) and without (d) $B_{0}=50 \mathrm{~T}$, respectively. The grey shaded regions mark the injection regions.

Because of the open bubble rear, the wake accelerating field $E_{z}$ has its maximum amplitude away from the laser axis, as shown in figure 2 (c). As a consequence, the most efficient acceleration region for the electrons moving along the bubble sheath is located at a distance away from the axis. Defining $\gamma_{\mathrm{rms}}, r_{\mathrm{rms}}$ and $z_{\mathrm{rms}}$ as the RMS Lorentz factor, radius and longitudinal position of energetic electrons $(\gamma>4)$ around the bubble rear, figure $2(\mathrm{~d})$ shows that both $\gamma_{\mathrm{rms}}$ and $r_{\mathrm{rms}}$ increase before the occurrence of electron injection. The increase of $\gamma_{\mathrm{rms}}$ can be attributed to the prolonged acceleration time in the expanding bubble along the density down-ramp. With increasing $\gamma_{\mathrm{rms}}$, however, $r_{\text {rms }}$ also increases because the centrifugal force $p_{\varphi} v_{\varphi} / r \propto \gamma_{\mathrm{rms}}$. As a result, finally, the increase of $r_{\text {rms }}$ inhibits electron injection.

To explain the suppression of electron injection through increasing $r_{\text {rms }}$, the instantaneous accelerating fields $E_{z}$ along the trajectories of two typical electrons are compared in figure 3(a). Firstly, we find that the injected electron \#1 that is closer to the axis experiences a stronger accelerating field than the non-injected electron \#2. Defining $\left|E_{\mathrm{z}, \max }\right|$ and $r_{\max }$ as the maximum of $E_{z}$ and the corresponding radial position where this maximum is achieved, figure $3(\mathrm{~b})$ shows a linear negative correlation between $\left|E_{z, \max }\right|$ and $r_{\max }$. This linear correlation is attributed to the linear distribution of $E_{z}$ along 
the transverse direction in the bubble regime [4], which is insensitive to the external magnetic field if $\omega_{c}<\omega_{p}$. Secondly, we find that the most efficient acceleration region (i.e., near the location of $\left|E_{z, \max }\right|$ ) for electron \#1 is much closer to its turning point than that for electron \#2. Therefore, the electron \#1 can stay in the efficient acceleration region for a longer period $\Delta T_{3}=11.2$, compared to $\Delta T_{1}=2.7$ for the electron $\# 2$ as show in figure 3(a). The stronger accelerating field and longer accelerating time combine to guarantee that electron \#1 can be accelerated to the bubble velocity and therefore be trapped. In contrast, electron \#2 can not be injected since it is further away from the axis. With increasing $r_{\text {rms }}$, more and more energetic electrons will be far away from the axis, as electron \#2. As a result, electron injection is inhibited for these electrons. Figure 3(c) shows that electron injection is triggered as soon as the increasing RMS velocity $\beta_{z, r m s}$ of energetic electrons exceeds the decreasing wake phase velocity along the density down-ramp. However, injection ends promptly because $\beta_{z}$ quickly decreases due to the increasing $r_{\text {rms }}$ for $B_{0}=50 \mathrm{~T}$. In contrast, figure $3(\mathrm{~d})$ shows that electron injection lasts a longer time since $\beta_{z}$ is nearly constant under $B_{0}=0$.

\subsection{Subfemtosecond electron bunches produced by 3D manipulation of the plasma bubble}

From the above analysis, it is evident that electron injection can be flexibly controlled by the combination of a density down-ramp and a magnetic field $B_{0}$. The density downramp triggers the electron injection, while the magnetic field $B_{0}$ suppresses the injection. The required $B_{0}$ to suppress the injection decreases with decreasing plasma density $n_{0}$ because the radius of the hole in the open bubble rear is inversely proportional to $n_{0}$ [38]. Figures $4(\mathrm{a})$ and $4(\mathrm{~b})$ illustrate that a weaker magnetic field $B_{0}=20 \mathrm{~T}$ can suppress injection for a lower density $n_{0}=0.0002 n_{c}$, where the corresponding length of injected electron bunch is reduced from $\sim 8$ to $\sim 1.5 \mu \mathrm{m}$. The required $B_{0}$ for suppressing injection can be further reduced by reducing the plasma density gradient. Figures 4(c) and 4(d) illustrate that electron injection can be effectively suppressed by $B_{0}=10 \mathrm{~T}$ if a more gentle down-ramp is adopted $(\alpha=0.3)$, which is also more feasible experimentally. Figures 4(e) and 4(f) show the phase-space distributions and currents of the ultrashort electron bunches presented in figures $4(\mathrm{~b})$ and $4(\mathrm{~d})$. The quasi-monoenergetic electron bunches have high peak currents $\sim 23.2(5) \mathrm{kA}$, respectively.

Table 1 lists the main parameters of injected electrons bunches with different magnetic field strengths $B_{0}$ and density fluctuations $\alpha$. It is seen that a magnetic field $B_{0}$ on the order of ten Tesla can significantly reduce the bunch duration, and a sub-femtosecond bunch can be achieved in the case with $\alpha=0.3$ and $B_{0}=10 \mathrm{~T}$. In the case $\alpha=0.3$, the electron injection can be completely suppressed by a magnetic field $B_{0}=20 \mathrm{~T}$. In general, it is found that the charge number decreases with the decreasing bunch duration. And the approximately linear relation between $Q$ and $\tau_{\text {rms }}[30,31]$ is still satisfied in these cases. In addition, the energy spread also decreases with the decreasing bunch duration since a shorter bunch can be loaded in a relatively narrower 

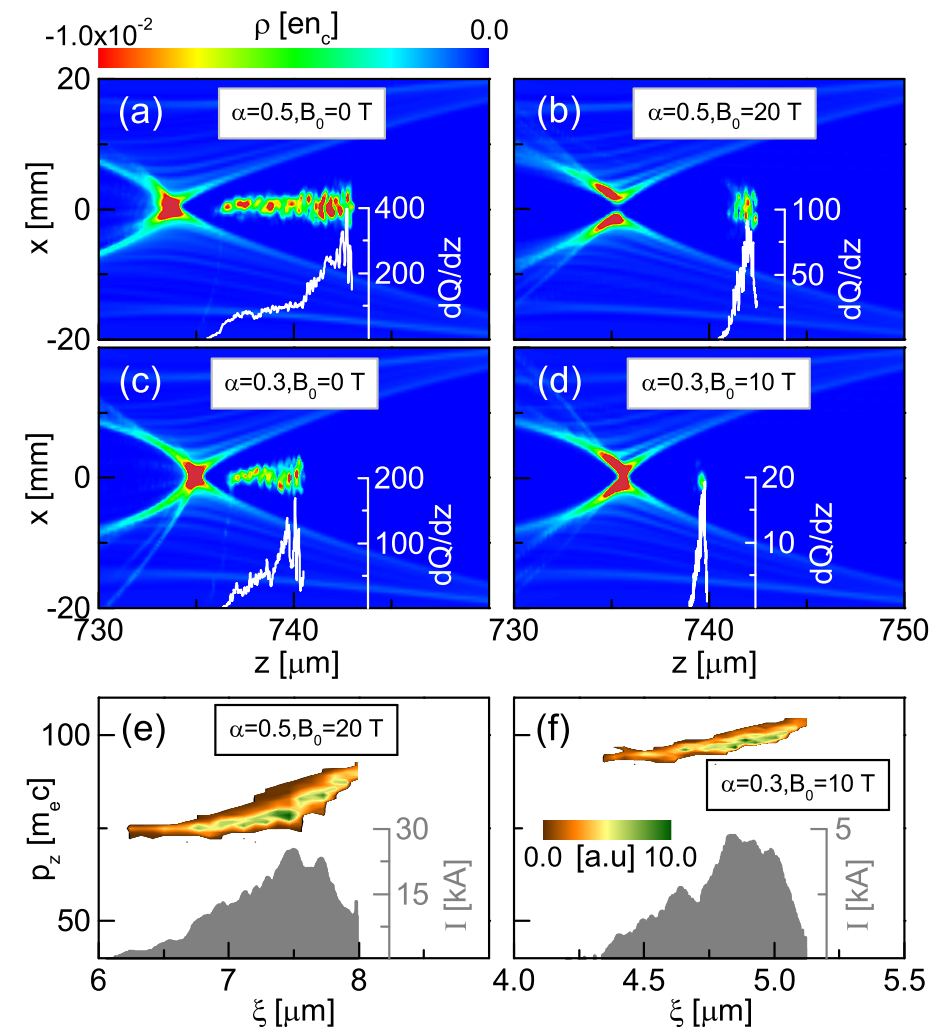

Figure 4. (Colour online) Bubble structures and injected electron bunches in densityprofile-tailored plasma with $n_{0}=0.0002 n_{c}, \sigma_{z}=100$ and (a) $B_{0}=0, \alpha=0.5$; (b) $B_{0}=20 \mathrm{~T}, \alpha=0.5$; (c) $B_{0}=0, \alpha=0.3$; (d) $B_{0}=10 \mathrm{~T}, \alpha=0.3$. Insets: Charge profiles of the injected electron bunches. (e)-(f) Phase-space distributions and currents of the injected electron bunches in (b) and (d), respectively.

Table 1. Charge number Q, RMS duration $\tau_{\text {rms }}$, transverse normalized emittance $\epsilon_{\mathrm{n}, \mathrm{x}(\mathrm{y})}$ and RMS energy spread $\Delta \mathrm{E}$ of injected electrons bunches at laser propagation distance $z \simeq 990 \mu \mathrm{m}$, subjected to a longitudinal magnetic field of different magnitudes $B_{0}$. The plasma density is tailored as $n_{e}=n_{0}\left[1+\alpha \exp \left(-\left(z-z_{i}\right)^{2} / 2 \sigma_{z}^{2}\right)\right]$ with $n_{0}=0.0002 n_{c}, z_{i}=350 \mu \mathrm{m}$, and $\sigma_{z}=100 \mu \mathrm{m}$. The results with different density fluctuations $\alpha=0.3$ and 0.5 are compared.

\begin{tabular}{c|c|cccc}
\hline$\alpha$ & $B_{0}[\mathrm{~T}]$ & $\mathrm{Q}[\mathrm{pC}]$ & $\tau_{\mathrm{rms}}[\mathrm{fs}]$ & $\epsilon_{\mathrm{n}, \mathrm{x}(\mathrm{y})}[\mu \mathrm{m}]$ & $\Delta \mathrm{E}[\mathrm{MeV}]$ \\
\hline \multirow{4}{*}{0.3} & 0 & 52.9 & 3.3 & $0.8(0.8)$ & 3.8 \\
& 10 & 7 & 0.6 & $0.8(1.1)$ & 1.3 \\
& 20 & 0 & - & - & - \\
\hline \multirow{3}{*}{0.5} & 0 & 119.4 & 6.2 & $0.8(1)$ & 5.6 \\
& 10 & 73.3 & 3.7 & $1.2(1.1)$ & 4 \\
& 20 & 35 & 1.3 & $1.2(1.3)$ & 2.4 \\
\hline
\end{tabular}

phase interval and hence feel an uniform accelerating field. However, the transverse emittance slightly increases in the cases $B_{0} \neq 0$ because the electron injection occurs off 
the axis around the bubble rear in these cases.

More important, our simulations confirm that the duration of electron bunch decreases monotonously with the increasing magnetic field $B_{0}$ for a given plasma density profile. This demonstrates that the longitudinal magnetic field provides an additional degrees of freedom in the control of electron injection besides the plasma density gradient. Therefore, our proposed method, introducing an external magnetic field into a density-tailored plasma, has more flexibility and power in the control of electron injection for the generation of ultrashort electron bunches.

\section{Conclusion}

In summary, sub-femtosecond electron bunches with a few $\mathrm{pC}$ in charge are accessible in the LWFA if electron injection is finely controlled by 3D manipulation of the plasma bubble. Combining a plasma density gradient with an external magnetic field, not only modulates the bubble velocity but also the electron longitudinal velocity. In this $3 \mathrm{D}$ manipulation, the increase of the bubble length along the density down-ramp increases the electron energy around the bubble rear, which results in electron injection, while an expanding hole in the bubble rear suppresses injection. The latter is attributed to the centrifugal force, which is proportional to the electron energy. The expanding hole will in return reduce the electron energy around the bubble rear. As a result, prompt suppression of electron injection is achieved. This 3D manipulation of the plasma bubble may enable realisation of sub-femtosecond electron bunches with readily accessible parameters both for density profiles and magnetic field strength. Furthermore, it may be extended to generate electron bunches with narrow energy spreads since the electrons can be properly phased in the wake and beam loading can be compensated as long as the electron injection is suppressed at a proper time [28].

\section{Acknowledgments}

We thank F. Y. Li for fruitful discussions. The work was supported by the National Basic Research Program of China (Grant No. 2013CBA01504), National Natural Science Foundation of China (Grant Nos. 11675108, 11774227, 11721091, and 11655002), National 1000 Youth Talent Project of China, UK Engineering and Physical Sciences Research Council (EPSRC) (Grant No. EP/N028694/1), the European Union's Horizon 2020 research and innovation programme under grant agreements 654148 LaserlabEurope and 653782 EuPRAXIA.

\section{References}

[1] Tajima T and Dawson J M 1979 Phys. Rev. Lett. 43267

[2] Esarey E, Schroeder C B and Leemans W P 2009 Rev. Mod. Phys. 811229

[3] Pukhov A and Meyer-Ter-Vehn J 2002 Appl. Phys. B 74355

[4] Kostyukov I, Pukhov A and Kiselev S 2004 Phys. Plasmas 115256 
[5] Lu W, Huang C, Zhou M, Tzoufras M, Tsung F S, Mori W B and Katsouleas T 2006 Phys. Plasmas 13056709

[6] Yi S A, Khudik V, Siemon C and Shvets G 2013 Phys. Plasmas 20013108

[7] Jaroszynski D A and Vieux G 2002 Adv. Accelerator Concepts 647 902; Clayton C E and Muggli P Eds. 2002 in 10th Workshop on Advanced Accelerator Concepts pp 902-913 Mandalay Beach Ca; Jaroszynski D A et al 2006 Phil. Trans. R. Soc. A 364689

[8] Rousse A, Phuoc K T, Shah R et al 2004 Phys. Rev. Lett. 93135005

[9] Schlenvoigt H P, Haupt K, Debus A et al 2008 Nat. Phys. 4130

[10] Fuchs M, Weingartner R, Popp A et al 2009 Nat. Phys. 5826

[11] Cipiccia S, Islam M R, Ersfeld B et al 2011 Nat. Phys. 7867

[12] Ersfeld B, Bonifacio R, Chen S, Islam M R, Smorenburg P W and Jaroszynski D A 2014 New J. Phys. 16093025

[13] Chen M, Luo J, Li F Y, Liu F, Sheng Z M and Zhang J 2016 Light Sci. Appl. 5 e16015

[14] Faure J, Rechatin C, Norlin A, Lifschitz A, Glinec Y and Malka V 2006 Nature 444737

[15] Lundh O, Lim J, Rechatin C 2011 Nat. Phys. 7219

[16] Kärtner F X, Ahr F, Calendron A L et al 2016 Nucl. Instrum. Methods Phys. Res. A 82924

[17] Dorda U, Assmann R, Brinkmann R et al 2016 Nucl. Instrum. Methods Phys. Res. A 829233

[18] Morimoto Y and Baum P 2018 Nat. Phys. $14252 \mathrm{M}$.

[19] Hassan M Th 2018 J. Phys. B: At. Mol. Opt. Phys. 51032005

[20] Buck A, Nicolai M, Schmid K, Sears C M S, Savert A, Mikhailova J M, Krausz F, Kaluza M C and Veisz L 2011 Nat. Phys. 7543

[21] Heigoldt M, Popp A, Khrennikov K, Wenz J, Chou S W, Karsch S, Bajlekov S I, Hooker S M and Schmidt B 2015 Phys. Rev. ST Accel. Beams 18121302

[22] Islam M R, Brunetti E, Shanks R P et al 2015 New J. Phys. 17093033

[23] Li F Y, Sheng Z M, Liu Y, Meyer-ter-Vehn J, Mori W B, Lu W and Zhang J 2013 Phys. Rev. Lett. 110135002

[24] Zeng M, Chen M, Sheng Z M, Mori W B and Zhang J 2014 Phys. Plasmas 21030701

[25] Mirzaie M, Li S, Zeng M et al 2015 Sci. Rep. 514659

[26] Bulanov S, Naumova N, Pegoraro F and Sakai J 1998 Phys. Rev. E 58 R5257

[27] Geddes C G R, Nakamura K, Plateau G R, Toth C, Cormier-Michel E, Esarey E, Schroeder C B, Cary J R and Leemans W P 2008 Phys. Rev. Lett. 100215004

[28] Gonsalves A J, Nakamura K, Lin C et al 2011 Nat. Phys. 7862

[29] Hansson M, Aurand B, Davoine X, Ekerfelt H, Svensson K, Persson A, Wahlström C G and Lundh O 2015 Phys. Rev. ST Accel. Beams 18071303

[30] Massimo F, Lifschitz A F, Thaury C and Malka V 2017 Plasma Phys. Control. Fusion 59085004

[31] Ekerfelt H, Hansson M, González I G, Davoine X and Lundh O 2017 Sci. Rep. 712229

[32] Martinez de la Ossa A, Hu Z, Streeter M J V, Mehrling T Kononenko J O, Sheeran B and Osterhoff J 2017 Phys. Rev. Accel.Beams 20091301

[33] Xu X L, Li F, An W, Dalichaouch T N, Yu P, Lu W, Joshi C and Mori W B 2017 Phys. Rev. Accel. Beams 20111303

[34] Tooley M P, Ersfeld B, Yoffe S R, Noble A, Brunetti E, Sheng Z M, Islam M R and Jaroszynski D A 2017 Phys. Rev. Lett. 119044801

[35] Hosokai T, Kinoshita K, Zhidkov A Maekawa A, Yamazaki A and Uesaka M 2006 Phys. Rev. Lett. 97 075004; Hosokai T et al 2010 Appl. Phys. Lett. 96121501

[36] Hur M S, Gupta D N and Suk H 2008 Phys. Lett. A 3722684

[37] Vieira J, Martinss S F, Pathak V B Fonseca R A, Mori W B and Silva L O 2011 Phys. Rev. Lett. 106 225001; Vieira J et al 2012 Plasma Phys. Control. Fusion 54124044

[38] Bulanov S V, Esirkepov T Zh, Kando M, Koga J K, Hosokai T, Zhidkov A G and Kodama R 2013 Phys. Plasmas 20083113

[39] Rassou S, Bourdier A and Drouin M 2015 Phys. Plasmas 22073104

[40] Zhao Q, Weng S M, Sheng Z M, Chen M, Zhang G B, Mori W B, Hidding B, Jaroszynski D A 
and Zhang J 2018 New J. Phys. 20063031

[41] Pollock B B, Froula D H, Davis P F et al 2006 Rev. Sci. Instrum. 77114703

[42] Fiksel G, Agliata A, Barnak D et al 2015 Rev. Sci. Instrum. 86016105

[43] Shi Y, Vieira J, Trines R M G M, Bingham R, Shen B F and Kingham R J 2018 Phys. Rev. Lett. 121145002

[44] Tsakiris G D, Gahn C and Tripathi V K 2000 Phys. Plasmas 73017

[45] Fonseca R A, Silva L O, Tsung F S et al 2002 Lect. Not. Comput. Sci. 2331342

[46] Kononenko O, Lopes N, Cole J et al 2016 Nucl. Instrum. Methods Phys. Res. Sect. A 829125 